\title{
BMJ Open A pragmatic health centre-based evaluation comparing the effectiveness of a PCV13 schedule change from $3+0$ to $2+1$ in a high pneumococcal carriage and disease burden setting in Malawi: a study protocol
}

Todd D Swarthout (D , ${ }^{1,2}$ Ana Ibarz-Pavon, ${ }^{2,3}$ Gift Kawalazira, ${ }^{4}$ George Sinjani, ${ }^{2}$ James Chirombo, ${ }^{2}$ Andrea Gori, ${ }^{1}$ Peter Chalusa, ${ }^{2}$ Farouck Bonomali, ${ }^{2}$ Roseline Nyirenda, ${ }^{2}$ Edwin Bulla ${ }^{2}$ Comfort Brown, ${ }^{2}$ Jacquline Msefula, ${ }^{2}$ Marjory Banda, ${ }^{5}$ Jean Kachala, ${ }^{4}$ Charles Mwansambo, ${ }^{4}$ Marc YR Henrion, $, 2,6$ Stephen B Gordon, ${ }^{2,6}$ Neil French, ${ }^{2,3}$ Robert S Heyderman (D) 1,2

To cite: Swarthout TD, IbarzPavon A, Kawalazira G, et al. A pragmatic health centrebased evaluation comparing the effectiveness of a PCV13 schedule change from $3+0$ to $2+1$ in a high pneumococcal carriage and disease burden setting in Malawi: a study protocol. BMJ Open 2021;11:e050312. doi:10.1136/ bmjopen-2021-050312

- Prepublication history for this paper is available online To view these files, please visit the journal online (http://dx.doi. org/10.1136/bmjopen-2021050312).

TDS and AI-P are joint first authors.

NF and RSH are joint senior authors.

Received 17 February 2021 Revised 20 April 2021 Accepted 27 April 2021

Check for updates

(C) Author(s) (or their employer(s)) 2021. Re-use permitted under CC BY. Published by BMJ.

For numbered affiliations see end of article.

Correspondence to

Todd D Swarthout;

t.swarthout@ucl.ac.uk

\section{ABSTRACT}

Introduction Streptococcus pneumoniae (the pneumococcus) is commonly carried as a commensal bacterium in the nasopharynx but can cause lifethreatening disease. Transmission occurs by human respiratory droplets and interruption of this process provides herd immunity. A 2017 WHO Consultation on Optimisation of pneumococcal conjugate vaccines (PCV) Impact highlighted a substantial research gap in investigating why the impact of PCV vaccines in low-income countries has been lower than expected. Malawi introduced the 13-valent PCV (PCV13) into the national Expanded Programme of Immunisations in 2011, using a $3+0$ ( 3 primary +0 booster doses) schedule. With evidence of greater impact of a $2+1$ (2 primary +1 booster dose) schedule in other settings, including South Africa, Malawi's National Immunisations Technical Advisory Group is seeking evidence of adequate superiority of a $2+1$ schedule to inform vaccine policy.

Methods A pragmatic health centre-based evaluation comparing impact of a PCV13 schedule change from 3+0 to 2+1 in Blantyre district, Malawi. Twenty government health centres will be randomly selected, with ten implementing a $2+1$ and 10 to continue with the $3+0$ schedule. Health centres implementing $3+0$ will serve as the direct comparator in evaluating $2+1$ providing superior direct and indirect protection against pneumococcal carriage. Pneumococcal carriage surveys will evaluate carriage prevalence among children 15-24 months, randomised at household level, and schoolgoers 5-10 years of age, randomly selected from school registers. Carriage surveys will be conducted 18 and 33 months following $2+1$ implementation.

Analysis The primary endpoint is powered to detect an effect size of $50 \%$ reduction in vaccine serotype (VT) carriage among vaccinated children 15-24 months old,

\section{Strengths and limitations of this study}

The study is supported by more than 20 years of routine pneumococcal surveillance.

- The study design is both pragmatic and robust, offering a methodology that is relevant to diverse settings in the region.

- By engaging community, institutional and government partners, we have optimised its relevance and increased the likelihood of community uptake.

- Limitations to this study include a risk of contamination between clusters (ie, children receiving a $2+1$ vaccine schedule who reside in or relocate to a cluster implementing the $3+0$ vaccine schedule or vice-versa).

expecting a $14 \%$ and $7 \% \mathrm{VT}$ carriage prevalence in the $3+0$ and $2+1$ arms, respectively.

Ethics and dissemination The study has been approved by the Malawi College of Medicine Research Ethics Committee (COMREC; Ref: P05.19.2680), the University College London Research Ethics Committee (Ref: 8603.002) and the University of Liverpool Research Ethics Committee (Ref: 5439). The results from this study will be actively disseminated through manuscript publications and conference presentations.

Trial registration number NCT04078997.

\section{INTRODUCTION}

Streptococcus pneumoniae, the pneumococcus, is commonly carried as a commensal bacterium in the nasopharynx but can cause lifethreatening disease. Infections due to the pneumococcus are estimated to be responsible for approximately 300000 deaths 
worldwide, with one-third of these occurring among children under 5 years of age and with the greatest burden in low- and middle-income country settings. ${ }^{12}$

S. pneumoniae has almost 100 serotypes, with nasopharyngeal (NP) carriage as a prerequisite for the development of disease but also a key process for developing natural immunity. ${ }^{3}$ Transmission occurs largely via human respiratory air droplets. With serotype-specific differences, carriage duration decreases with age, lasting from 2 weeks in adults up to 4 months among children. ${ }^{45}$ Pneumococcal carriage prevalence is age-dependent, peaking among children $<5$ years. Pneumococcal carriage prevalence reported in subSahara Africa are among the highest described; up to $28 \%$ among adults and in excess of $80 \%$ in children $<5$ years old, resulting in high transmission rates. ${ }^{6-14}$

In HICs, routine administration of pneumococcal conjugate vaccines (PCV) through the infant immunisation schedule has contributed to a rapid decline of vaccine serotype invasive pneumococcal disease (VT-IPD) in both vaccinated and unvaccinated populations. ${ }^{15-23}$ $\mathrm{PCVs}$ protect the vaccinated individual (direct protection) against pneumococcal disease and carriage. The resulting reduction in carriage also interrupts transmission from the vaccinated individual to the unvaccinated population (indirect protection). The resulting herd immunity effect has been a major contributor to the success of vaccination programmes. ${ }^{24-26}$ The added costeffectiveness and vaccine impact gained through indirect protection have been key drivers of vaccination policy in these settings. ${ }^{27} 28 \mathrm{PCV}$ impact on pneumococcal carriage continues to be considered a viable endpoint in vaccine licensure evaluations. ${ }^{29}$

Studies undertaken prior to PCV introduction in The Gambia, Kenya, Mozambique, Malawi and South Africa reported VT carriage prevalence ranging from $28 \%$ to nearly $50 \%$ among children $<5$ years old. ${ }^{30-33}$ While PCV introduction in African countries has resulted in substantial direct effects in reducing risk of VT-IPD, ${ }^{34}$ pneumonia and all-cause mortality ${ }^{35}$ among vaccinated children, ${ }^{10} 1136$ the impact of PCV on VT carriage has been markedly less than that observed in HICs. Although Kenya, ${ }^{12}$ the Gambia, ${ }^{13}$ Mozambique ${ }^{14}$ and South Africa ${ }^{31}$ have reported VT carriage reductions, prevalence remains higher than expected, and serotype replacement (ie, rise in non-VT carriage prevalence associated with decrease in VT carriage) is increasing. ${ }^{67}$ As the impact of PCV on carriage is considered an indicator of vaccine impact, ${ }^{29}$ it remains uncertain whether PCV introduction in subSahara Africa will achieve the sustained direct or indirect protection necessary to reduce pneumococcal carriage to levels sufficient to interrupt transmission and disease. ${ }^{38}$

Currently, WHO recommends the implementation of the PCV vaccine using either a $3+0$ schedule (three primary doses, most commonly at 6,10 , and 14 weeks of age) or a $2+1$ schedule (two primary infancy doses at 6 and 14 weeks of age and one booster at 9 months of age or after the first year of life). The WHO further recommends that the decision on which schedule to use be based on the epidemiology of disease in the local setting. ${ }^{39}$ While both schedules have been shown to be effective in reducing VT disease and VT carriage, there have been no direct comparisons of vaccine impact on carriage in a high burden setting. ${ }^{40}$

A 2017 WHO Technical Expert Consultation on Optimisation of PCV Impact highlighted a substantial research gap in investigating why the impact of PCV vaccines in low-income countries has been less than expected, underlining the need to define an optimal PCV vaccination schedule that will maximise their benefit in such settings. ${ }^{41}$ The PCV Review of Impact Evidence, commissioned to supplement the WHO Expert Consultation, is a systematic review of available evidence on PCV effectiveness and impact on NP carriage, disease and mortality, as well as on PCV immune response. The review included evidence from research studies published between January 2010 and December 2016 recorded in 14 databases. Those attending the 2017 WHO Technical Expert Consultation gave head-to-head studies of $2+1$ vs $3+0$ vaccine schedules the greatest research priority. ${ }^{42}$ Countries with a high pneumococcal disease and carriage burden, such as Malawi, could implement a $2+1$ schedule quickly, with limited logistical or financial demands, providing a booster dose at the time of the first measles vaccine at 9 months of age.

Malawi introduced the 13-valent PCV (PCV13) into the national Expanded Programme of Immunisations (EPI) in November 2011, using a $3+0$ schedule $(6,10$ and 14 weeks of age), with a three-dose catch-up vaccination campaign among all infants $<1$ year of age. This introduction has been highly successful, with field studies showing an EPI vaccine coverage exceeding 90\%. ${ }^{43}$ Similar to other settings, vaccine introduction resulted in a 70\% reduction in IPD among PCV-vaccinated children $^{34}$ and an estimated $35 \%$ fall in all-cause mortality. ${ }^{35}$ However, high levels of residual VT carriage persist in Malawi among vaccinated children up to 8 years after the introduction of the vaccine. ${ }^{45}$ As presented by Lourenço et $a t^{46}$ a high force of infection in settings such as Malawi contribute significantly to a $3+0$ schedule achieving only a short duration of VT carriage control in infants. Though a 2+1 schedule, as implemented in South Africa, may improve colonisation control, this remains unproven in other African settings such as Malawi.

In this context, the Malawi Ministry of Health $(\mathrm{MoH})$ and the National Immunisations Technical Advisory Group are now seeking evidence of adequate superiority of a $2+1$ vaccine schedule to inform a change to Malawi's current EPI schedule. To this aim, a pragmatic health centre-based evaluation comparing the current $3+0$ schedule to a $2+1$ schedule will be implemented in Blantyre District, southern Malawi. Two pneumococcal carriage surveys, conducted 18 and 33 months following the implementation of the $2+1$ schedule, will have the objective of comparing the effect of the two schedules against carriage reduction among otherwisehealthy children and evaluate their potential to enhance herd immunity. 


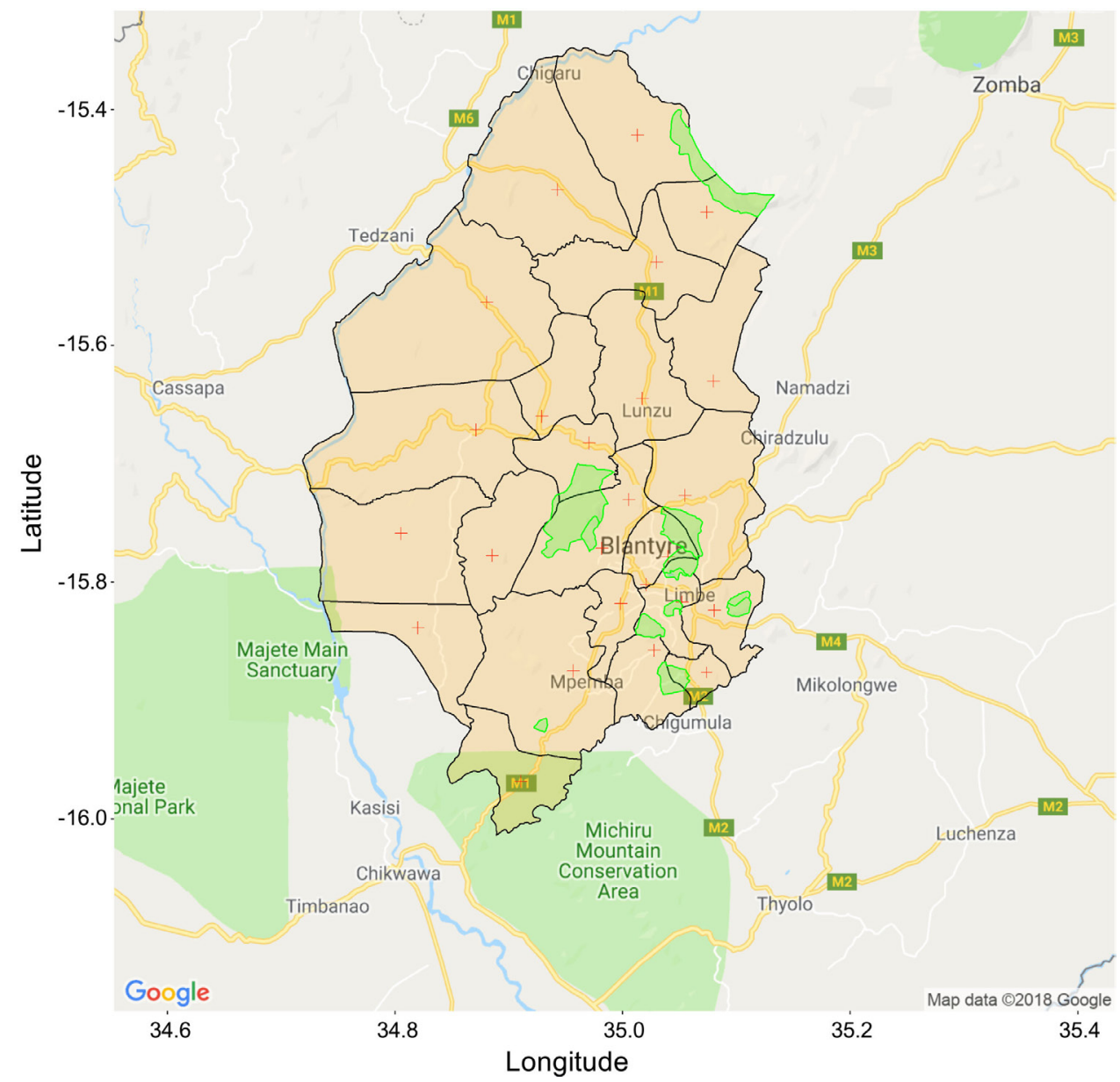

Figure 1 Map of Blantyre district with borders of health centre catchment areas. Red crosses (+) indicate location of health centres offering EPI vaccination. Areas shaded in green are non-inhabited (incl. mountains, industrial zones and other regions administratively declared not for habitation). EPI, Expanded Programme of Immunisations.

\section{METHODS AND ANALYSIS}

\section{Study design}

The study is a pragmatic health centre-based randomised evaluation of the direct effect of a 2+1 PCV13 vaccine schedule on pneumococcal carriage in vaccinated infants and the indirect effect on non-vaccine eligible children and high-risk adults.

\section{Study setting}

The study will be conducted in Blantyre district, southern Malawi. The District is $240 \mathrm{~km}^{2}$. Healthcare is delivered through a network of private and government hospitals and 28 government primary health centres, where EPI vaccinations are administered. Queen Elizabeth Central Hospital (QECH) is the government referral hospital providing free medical care to the 1.3 million urban, periurban and rural residents of Blantyre District. Children $<5$ years account for $16 \%$ of the total population. ${ }^{47}$ Health centres cover a fixed geographic area (here referred to as 'clusters'), and study sampling will be undertaken within these clusters (figure 1).

\section{Study questions}

We will investigate whether a 2+1 PCV13 vaccination schedule (two primary doses at 6 and 14 weeks of age +1 booster dose at 9 months of age), compared with the current $3+0$ schedule (three primary doses at 6, 10 and 14 weeks of age with no booster), is superior in reducing VT carriage prevalence among vaccinated children and, consequently, creating a superior herd effect among older children and HIV-infected adults. To assess the effect of a district-wide change from $3+0$ to $2+1$, this study will address two specific research questions: First, will a 2+1 schedule provide enduring vaccine-induced protection against pneumococcal VT carriage into the second year of life? Second, will the $2+1$ vaccine schedule generate stronger herd protection and result in decreased VT carriage in older children and unvaccinated high-risk adults in the general population?

\section{Primary objective}

The primary objective of the study is to evaluate the direct effect of a 2+1 PCV13 vaccination schedule on VT pneumococcal carriage among children aged 15-24 months, 3 years after introducing the $2+1$ schedule. This objective will answer the question of whether the $2+1$ schedule induces a more enduring direct protection against pneumococcal VT carriage into the second year of life, compared with that observed with a $3+0$ schedule. 


\section{Secondary objectives}

The secondary objectives of the study are to evaluate, 3 years after introducing the $2+1$ schedule, (1) the indirect effect of a 2+1 schedule on VT pneumococcal carriage among children aged 5-10 years (PCV age-ineligible at time of implementing the $2+1$ schedule); (2) the indirect effect of a $2+1$ vaccination schedule on VT pneumococcal carriage among HIV-infected adults 18-40 years old and on antiretroviral therapy (ART).

To address these questions, the Malawi MoH and Blantyre District Health Office (DHO) will randomly select 10 health centres (among a total 28 in Blantyre District) in which the routine PCV13 schedule will be switched to a $2+1$ schedule. An additional 10 health centres will be randomly selected to continue with the current $3+0$ schedule but will serve as the direct comparator in evaluating the effectiveness of the $2+1$ schedule.

\section{A pragmatic study design}

This switch to a $2+1$ schedule is an initiative led by the $\mathrm{MoH}$ and will be implemented within the scope of the routine EPI programme, subject to EPI standard procedures for delivery, monitoring and performance assessment. Implementation of all vaccination activities will be implemented, as per routine practices, by MoH EPI vaccinators through the routine EPI programme. The $\mathrm{MoH}$ will monitor completeness of dosing following standard reporting practices within the scope of the EPI. Routine study activities throughout the duration of the 3-year study period will include research nurses providing support and guidance to the EPI vaccination teams through weekly site visits. In addition, research enumerators will monitor patient-retained health passports of a representative sample population of vaccinees to confirm they are receiving the proper vaccine schedule $(2+1$ or $3+0)$ assigned to the catchment population of their respective health centre.
The pragmatic design of this study has been assessed through the tool The PRagmatic-Explanatory Continuum Indicator Summary 2 (PRECIS-2). ${ }^{48}$ This tool was developed to help trialists make design decisions consistent with the intended purpose of their trial. To facilitate domain discussion and consensus, PRECIS-2 assesses the pragmatic design through nine domains-eligibility criteria, recruitment, setting, organisation, flexibility (delivery), flexibility (adherence), follow-up, primary outcome and primary analysis-scored from 1 (very explanatory) to 5 (very pragmatic). The report from this tool is in the format of a wheel (figure 2). Results (mean score: 4.4 and range: 3-5) supports that this is largely a pragmatic randomised study undertaken in the 'real world' and with usual care and is intended to help support a decision on whether to deliver an intervention.

\section{$3+1$ schedule during early period of initiation}

To ensure the $2+1$ schedule is implemented efficiently, and to limit the risk of confusion during the transition to the $2+1$ schedule (including not deferring PCV13 vaccination to a later visit), a $3+1$ schedule will be implemented during the first several months at health centres implementing the $2+1$ schedule. At these health centres, children who have received either their 10 weeks or 14 weeks PCV13 dose before $2+1$ implementation will receive a dose at their 14weeks (third dose PCV13) and their 40 weeks (fourth dose PCV13). Approximately 6 months after 2+1 implementation, all first-contact vaccine visits (post $2+1$ implementation) will be for the scheduled visit at 6 weeks of age and the $3+1$ schedule will no longer be required.

\section{Carriage surveys}

The study will include two cross-sectional carriage surveys, implemented 18 and 33 months after the switch to $2+1$. Carriage surveys will be conducted using a wellestablished methodology implemented extensively in

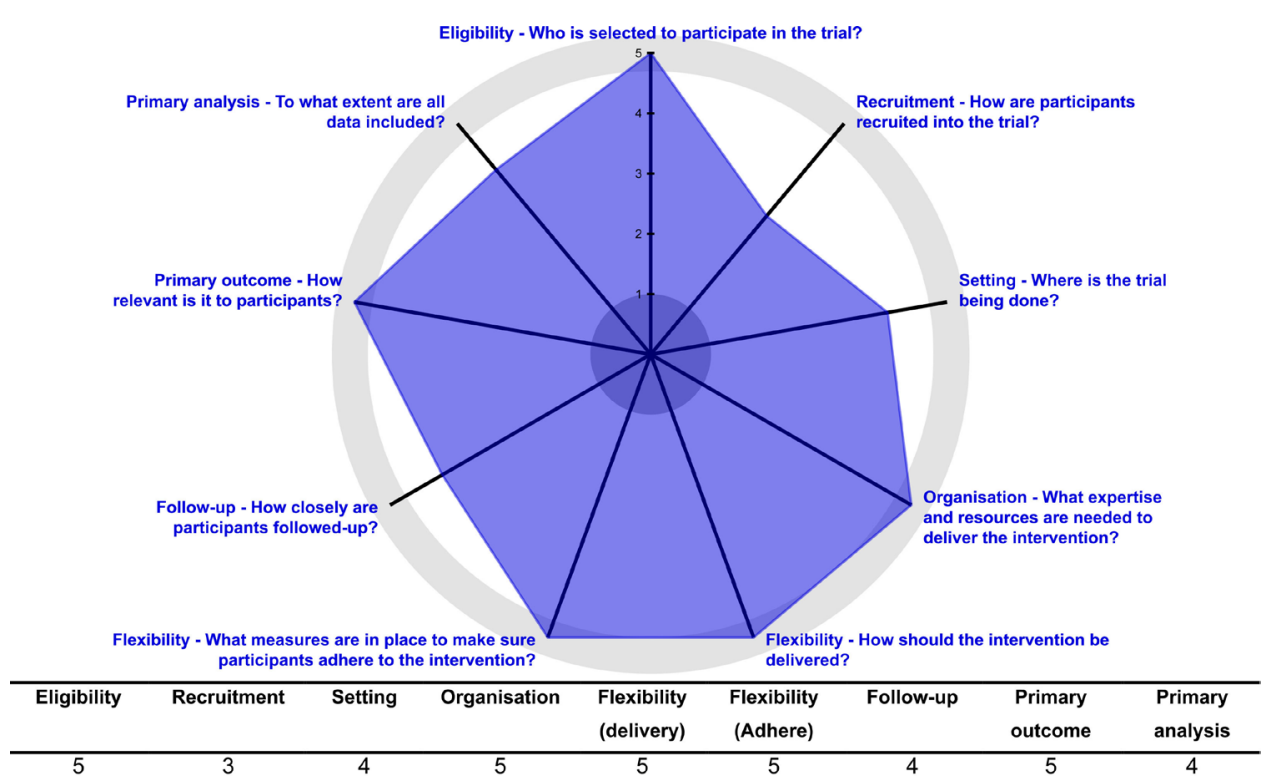

Figure 2 The PRagmatic-Explanatory Continuum Indicator Summary 2 wheel with study-specific scores. 


\section{Surveillance Sampling Frame}

\begin{tabular}{|c|c|c|c|}
\hline $\begin{array}{c}\text { Children } \\
15-24 \text { months old } \\
\text { PCV13 immunized } 3+0 \text { vs. } 2+1 \\
\text { from randomized clusters } \\
(\mathrm{N}=800 / \text { survey })\end{array}$ & $\begin{array}{c}\text { Children } \\
9 \text { months old } \\
\text { PCV13 } 3+0 \text { or } 2+0 \text { immunized } \\
\text { from randomized clusters } \\
(\mathrm{N}=800 \text { for } 1 \text { survey })\end{array}$ & $\begin{array}{c}\text { Children } \\
6-10 \text { years old } \\
\text { PCV13 } 3+0 \text { immunized } \\
\text { from randomized clusters } \\
(\mathrm{N}=750 \text { / survey })\end{array}$ & $\begin{array}{c}\text { Adults, HIV-infected on ART } \\
18-40 \text { years old } \\
\text { PCV13 unimmunized } \\
\text { from QECH ART Clinic } \\
(\mathrm{N}=1,200 \text { over study period })\end{array}$ \\
\hline 1 & I & 1 & 1 \\
\hline $\begin{array}{l}\text { Household Recruitment } \\
\text { Stratified random sampling }\end{array}$ & $\begin{array}{l}\text { Health Centre Recruitment } \\
\text { Convenience sampling }\end{array}$ & $\begin{array}{l}\text { School Recruitment } \\
\text { Stratified random sampling }\end{array}$ & $\begin{array}{l}\text { QECH ART Clinic } \\
\text { Systematic sampling }\end{array}$ \\
\hline I & I & $\downarrow$ & I \\
\hline $\begin{array}{l}2 \text { Carriage Surveys } \\
18 \mathrm{~m} \& 33 \mathrm{~m} \text { after } 2+1 \\
\text { implemented }\end{array}$ & $\begin{array}{l}1 \text { Carriage Survey } \\
9 \mathrm{~m} \text { after } 2+1 \\
\text { implemented }\end{array}$ & $\begin{array}{l}2 \text { Carriage Surveys } \\
18 \& 33 \mathrm{~m} \text { after } 2+1 \\
\text { implemented }\end{array}$ & $\begin{array}{l}\text { Rolling Carriage Survey }{ }^{* *} \\
\text { Starting } 18 \mathrm{~m} \text { after } 2+1 \\
\text { implemented }\end{array}$ \\
\hline 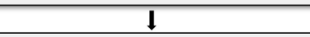 & I & $\downarrow$ & 1 \\
\hline \multicolumn{4}{|c|}{ Per Nasal Swab } \\
\hline I & \multicolumn{3}{|c|}{ I } \\
\hline $\begin{array}{l}\text { Primary end-point } \\
\text { VT carriage prevalence } \\
\text { 3yrs after } 2+1 \text { implemented: }\end{array}$ & \multicolumn{3}{|c|}{$\begin{array}{l}\text { Secondary end-point } \\
\text { VT carriage prevalence } \\
3 \text { years after } 2+1 \text { implemented }\end{array}$} \\
\hline $\begin{array}{l}\text { Interim analysis after survey } 1 \\
3+0 \text { vs. } 2+1 \text { clusters }^{\star}\end{array}$ & \multicolumn{3}{|c|}{$\begin{array}{l}\text { * } 50: 503+1 / 2+1 \text { sampling in } 2+1 \text { clusters to maximise comparativeness with primary endpoint } \\
{ }^{*} \text { Rolling survey logistically most effective in an ART clinic setting (stable baseline) }\end{array}$} \\
\hline
\end{tabular}

Figure 3 Carriage surveillance sampling frame black dashed vertical line separates primary (left of dashed line) from secondary study objectives. ART, antiretroviral; M, months; PCV, pneumococcal conjugate; QECH, Queen Elizabeth Central Hospital.

the setting, ${ }^{45} 46$ allowing comparability of study results to previous carriage surveys conducted in the area. The sampling frame is illustrated in figure 3 .

Sampling will include:

1. Children 15-24 months of age, PCV13-vaccinated (confirmed by reviewing patient-retained health passports) with either the $2+1$ or the $3+0$ schedule. Evaluation within this age group will allow us to evaluate for our primary objective, evaluating the direct effect of vaccination on carriage. Within each of the catchment areas of the 20 participating health centres, we will conduct a random walk method to systematically identify households with eligible children. A 'egg and yolk' strategy will be applied, defining two geographic sampling perimeters around each health centre. Sampling will prioritise those living within the geographic perimeter closest to the health centre. In the event that recruitment targets are not met, sampling will move into the second geographic perimeter. This strategy will maximise the likelihood of recruiting children who have received their vaccines at the selected health centre, minimising recruitment from buffer zonal borders and therefore minimising risk of contamination (ie, recruitment of children receiving a PCV13 vaccine schedule different than the schedule assigned to the child's local health centre).

2. Children 5-10 years of age, PCV13-vaccinated with the $3+0$ schedule. These children, having received PCV13 at infancy, will be recruited from government schools. Evaluation within this age group will allow us to evaluate for a secondary objective, evaluating the indirect effect of a 2+1 schedule on VT pneumococcal carriage among children aged 5-10 years, the majority of whom received PCV13 infancy EPI vaccination using a $3+0$ schedule. Six schools will be selected, three located centrally in each of the $3+0$ clusters and the $2+1$ clusters. Children will be chosen at random from school registers. This component will allow the evaluation of indirect effects of the $2+1$ compared with $3+0$ schedule among children with waning vaccine-induced immunity.

3. Adults 18-40 years of age, HIV-infected on ART and PCV13-unvaccinated. Evaluation within group will allow us to achieve the secondary objective of evaluating the indirect effect of a $2+1$ schedule on VT pneumococcal carriage among HIV-infected adults (18-40 years) on ART, a population at high risk of pneumococcal disease. Participants will be recruited from the QECH, Lighthouse ART Clinic in Blantyre.

\section{Safety}

To assess the differential effects of 3 vs 2 primary vaccine doses in the first year of life, an additional carriage survey will be implemented at health centres approximately 9 months after $2+1$ implementation. A convenience sample of children aged 9 months will be recruited at health centres providing either the $2+1$ or $3+0$ schedule. This will allow an evaluation of carriage prevalence among children receiving 3 (according to the $3+0$ schedule) vs 2 (according to the $2+1$ schedule and presenting for their booster dose) primary doses. Additionally, this additional carriage survey will contribute to evaluating the coverage of the 2+1 vaccination schedule and the proportion of children receiving all three PCV13 doses at health centres providing the $2+1$ schedule.

\section{Inclusion and exclusion criteria}

Inclusion criteria for all individuals recruited includes permanent resident in Blantyre District. Among children 15-24 months of age (assigned to either $2+1$ or $3+0$ schedule), inclusion criteria additionally include: aged between 15 and 24 months, parent/legal guardian providing written informed consent, evidence (recorded in health passport) of having received a full schedule 
of PCV13. Among children 5-10 years of age, inclusion criteria include: aged between 5-10 years, parent/legal guardian providing written informed consent, providing written informed assent (if the child is aged $\geq 8$ ), and either verbal or documented evidence of having received PCV13. Among adults, inclusion criteria include: aged 18-40 years, providing written informed consent, and being HIV-infected and receiving ART.

Among children 9 months of age, recruited as part of the safety component, inclusion criteria include: aged 9 months, parent/legal guardian providing written informed consent, and evidence (recorded in health passport) of having received either a full 3+0 PCV13 vaccine schedule (health centres implementing $3+0$ ) or both primary doses of PCV13 at approximately 6 and 14 weeks of age (health centres implementing $2+1$ ).

Exclusion criteria for all screened individuals include receiving TB treatment at time of screening, hospitalisation for pneumonia within 14 days prior to study screening and terminal illness. Exclusion criteria for all children include parental/legal guardian not providing consent, not providing assent (for children aged $\geq 8$ years), having received antibiotic treatment within 14 days prior to study screening. Exclusion criteria for adults include not providing written informed consent or prior vaccination with a pneumococcal vaccine.

\section{Intervention}

The intervention will consist of two carriage surveys conducted 18 and 33 months after the 10 randomised health centres switch from a 3+0 to a 2+1 PCV13 schedule. In both surveys, a single NP swab will be collected from each participant. Following previously described WHOrecommended procedures, ${ }^{45}$ these will be collected and taken to the Malawi-Liverpool-Wellcome Trust Clinical Research Programme laboratory in Blantyre for the isolation and characterisation of S. pneumoniae. VT pneumococcal carriage will be serotyped by latex agglutination. Samples from participants with confirmed pneumococcal carriage will be sent to the UK for assessment of multiple serotype carriage (genomic microarray) and for whole genome sequencing.

\section{Expected outcomes}

The primary endpoint of this study will be the difference in VT pneumococcal carriage prevalence among children aged 15-24 months, comparing those vaccinated with PCV13 in either a $2+1$ or $3+0$ schedule, 3 years after implementing the switch to a $2+1$ schedule. Additionally, the study will evaluate four secondary outcomes: (1) difference in VT carriage prevalence among children 5-10 years old, 18 months and 33 months after $2+1$ implementation, (2) VT carriage prevalence among HIV-infected adults aged 18-40 years and receiving ART at the time of sampling, (3) VT carriage prevalence among infants aged 9 months, who will have received three primary doses $(3+0$ health centres $)$ or two primary doses $(2+1$ health centres) prior to the booster dose, 9 months after the implementation of the $2+1$ schedule and (4) prevalence of multiple serotype carriage, 18 and 33 months after $2+1$ implementation

\section{Patient and public involvement statement}

Prior to development of the protocol, key stakeholders were informed of the study, including the study sites (ie, selected health centres) and their surrounding communities (ie, catchment areas), the $\mathrm{DHO}, \mathrm{MoH}$ and the Ministry of Education. We actively sought and incorporated input from these stakeholders into the study objectives and overall design. Community sensitisation will be further strengthened through a community advisory board.

\section{Statistical methods}

\section{Study power and sample size calculation}

The primary endpoint is powered to detect an effect size of $50 \%$ reduction in VT carriage, expecting a $14 \%$ and $7 \%$ VT carriage prevalence among vaccinated children 15-24 months old in the $3+0$ and $2+1$ arms, respectively. Sample sizes were calculated based on a power of $80 \%$ and a statistical significance of 0.05 . The calculations accounted for household similarities using an intraclass correlation (ICC) of 0.005 (based on previous experience in this setting) and adjusted for a design effect (dependent on both ICC and cluster size.) of 1.21. Minimum sample sizes needed to achieve the necessary power under these assumptions are shown in table 1.

To assess the primary and secondary endpoints (1) One child 15-24 months of age $(2+1$ or $3+0)$ will be recruited from each of 40 households randomly selected within each of the 20 clusters. This is a total 800 vaccinated children per survey (1600 total for two surveys). (2) A total of 125 children will be recruited from each of the six schools per survey. This is a total 750 children per survey (1500 total). (3) A total of $1200 \mathrm{HIV}$-infected adults will be recruited from the QECH ART Clinic over the course of the 3-year study period. (4) A total of 800 children 9 months of age will be recruited from the vaccination centres 9 months after schedule change (800 total).

\section{Data collection, management and anonymisation procedures}

Demographic data and relevant medical history will be collected using password-protected electronic data capturing. Each participant will be assigned a unique participant identification number (PID) at recruitment. This PID will be used in all datasheets and files, and will be linked to the laboratory data, hence, only anonymised data will be used for the analysis. Fully anonymised data will be uploaded daily to a secured on-site server, which is backed up daily to both local and off-site facilities. A logbook containing identifiable information (including name) will be kept separate in a secured location by an authorised member of the study team and will only be accessed by authorised study members. This will allow the study team to recover any missing epidemiological information at a later date (eg, missing vaccination dates) and to facilitate any participants who choose to withdraw consent at any time. 


\begin{tabular}{lllllllllll}
\hline \multicolumn{2}{l}{ Table 1 } & Sample size estimations & & & & & & \\
\hline N per cluster & ICC & $\mathbf{p 1}$ & $\mathbf{p 2}$ & Power & Alpha & DE & N per arm & Clusters, per arm & Clusters, total & N total \\
\hline 40 & 0.005 & 0.14 & 0.07 & 0.8 & 0.05 & 1.20 & 358 & 9 & 18 & 720 \\
40 & 0.005 & 0.14 & 0.07 & 0.8 & 0.05 & 1.21 & 400 & 10 & 20 & 800 \\
45 & 0.005 & 0.14 & 0.07 & 0.8 & 0.05 & 1.22 & 366 & 9 & 18 & 810 \\
50 & 0.005 & 0.14 & 0.07 & 0.8 & 0.05 & 1.25 & 373 & 8 & 16 & 800 \\
55 & 0.005 & 0.14 & 0.07 & 0.8 & 0.05 & 1.27 & 381 & 7 & 14 & 770 \\
60 & 0.005 & 0.14 & 0.07 & 0.8 & 0.05 & 1.30 & 388 & 7 & 14 & 840 \\
40 & 0.01 & 0.14 & 0.07 & 0.8 & 0.05 & 1.39 & 417 & 11 & 22 & 880 \\
45 & 0.01 & 0.14 & 0.07 & 0.8 & 0.05 & 1.44 & 432 & 10 & 20 & 900 \\
50 & 0.01 & 0.14 & 0.07 & 0.8 & 0.05 & 1.49 & 447 & 9 & 18 & 900 \\
55 & 0.01 & 0.14 & 0.07 & 0.8 & 0.05 & 1.54 & 462 & 9 & 18 & 990 \\
60 & 0.01 & 0.14 & 0.07 & 0.8 & 0.05 & 1.59 & 477 & 8 & 16 & 960 \\
40 & 0.015 & 0.14 & 0.07 & 0.8 & 0.05 & 1.59 & 475 & 12 & 24 & 960 \\
\hline
\end{tabular}

$\mathrm{DE}$, design effect; ICC, intraclass correlation.

\section{Statistical analyses}

Continuous variables will be summarised by means and $\mathrm{SD}$, or medians and IQRs if the distribution exhibits skew. Categorical variables will be summarised by frequency distributions. Direct effects of the PCV13 schedule change on VT carriage will be ascertained by comparing carriage prevalence in children aged 15-24 months residing in the recruitment clusters of health centres randomised to the $2+1$ schedule and those in recruitment clusters of health centres randomised to the $3+0$ schedule. Indirect effects will be evidenced by comparing children in the recruitment clusters of health centres randomised to the $3+0$ schedule among older children (5-10 years) and HIV +adults. Additionally, data obtained in this study will be compared with those obtained from previous carriage surveys to ascertain any changes in VT carriage prevalence before and after the PVC13 schedule change. Statistical tests will be selected depending on the distribution patterns of the data. Potential confounders and sources of interaction (including age, gender and health centre) will be identified by testing the association between variables and VT carriage and included in the multivariable models when $\mathrm{p}<0.1$. Sensitivity analyses will include assessing impact of (1) receiving only one, only two or all three doses PCV; (2) having document-confirmed PCV vaccination or (3) schedule adherence to within 2 weeks of each scheduled dose on VT prevalence and on the VT distribution.

\section{ETHICS AND DISSEMINATION Ethics approvals}

The study has been approved by the Malawi College of Medicine Research Ethics Committee (COMREC; Ref: P05.19.2680), the University College London Research Ethics Committee (Ref: 8603.002) and the University of Liverpool Research Ethics Committee (Ref: 5439).

\section{Data monitoring external advisory group}

The implementation of the study protocol will be reviewed and monitored by an External Advisory Group, providing oversight of the study activities and advise the study team at the time of the interim and final analyses. The group will include experts from the University of Malawi, College of Medicine, the London School of Hygiene \& Tropical Medicine and the Medical Research Council.

\section{Interim analysis and changes to public health policy}

On completion of the first carriage survey, an interim analysis will be implemented using data obtained on the VT carriage prevalence among vaccinated children 15-24 months of age. These results will be used to assess possible adaptation of the second community carriage survey. Three possible scenarios are considered: (1) If convincing evidence of major change in carriage prevalence is demonstrated, immediate action will be discussed with the $\mathrm{MoH}$ to move to change the schedule and adapt year-3 sampling; (2) If no major change in carriage prevalence is identified, the study will continue as planned; (3) If carriage prevalence has fallen by more than $30 \%$ but does not meet the primary threshold of $50 \%$, the second carriage survey will be brought forward by 6 months. Decisions will be reached in consensus between investigators, the Expert Advisory group, and the $\mathrm{MoH}$.

\section{Informed consent process}

The study will only recruit children whose parents/legal guardians have the capacity to provide informed consent or, in the case of adults, are capable of giving consent. Children who are minors but $\geq 8$ years of age will be required to provide informed assent, in addition to parents/legal guardians providing informed consent. Participants will receive both verbal and written information about the study and will be given the opportunity to ask questions and express their doubts and concerns before accepting 
to take part. They will also be given time to reflect before they come to a decision. An informed consent and/or assent form will be signed and dated by the participant and a member of the research team. The participant will keep a copy of the document, and a second one will be kept in the study file with the Principal Investigator based in Blantyre, Malawi. Participants will be informed of their right to withdraw consent at any point until study ends without the need to provide a reason and without penalty.

\section{DISSEMINATION POLICY AND PLANS}

Study results will be shared with local stakeholders and published in peer-reviewed journals. Partial results and interim analyses will be shared with the Malawi $\mathrm{MoH}$ and other relevant policymakers and decision-making stakeholders. Partial and final findings will be presented at relevant international conferences and meetings. Copies of all published materials and reports will be shared with the research ethics committees and collaborators. We will return to the community partners and work with the community advisory board to report and further disseminate our results into those communities where we worked.

\section{Author affiliations}

${ }^{1} \mathrm{NIHR}$ Global Health Research Unit on Mucosal Pathogens, Division of Infection and Immunity, University College London, London, UK

${ }^{2}$ Malawi-Liverpool-Wellcome Trust Clinical Research Programme, University of

Malawi College of Medicine, Blantyre, Malawi

${ }^{3}$ Centre for Global Vaccine Research, Institute of Infection and Global Health, University of Liverpool Faculty of Health and Life Sciences, Liverpool, UK

${ }^{4}$ Ministry of Health, Lilongwe, Malawi

${ }^{5}$ Ministry of Education, Blantyre, Malawi

${ }^{6}$ Department of Clinical Sciences, Liverpool School of Tropical Medicine, Liverpool, UK

Acknowledgements The authors thank the MLW laboratory management team, led by Brigitte Denis and George Selemani.

Contributors Contributors TDS, GK, CM, NF and RSH conceived the study. TDS, GK, $\mathrm{CM}, \mathrm{NF}$ and RSH designed the study with contributions from Al-P, JC, JK, MB, MH and SBG. TDS, CB and JM oversaw development of laboratory methods. TDS, Al-P, $A G, P C, F B, R N, E B$ and GS designed study documents. TDS and Al-P jointly wrote the first draft. All authors have commented on the manuscript. All authors have read and approved the final manuscript.

Funding This work was funded by Bill \& Melinda Gates Foundation, USA (Ref: OPP1185516) to RSH; National Institute for Health Research (NIHR) Global Health Research Unit on Mucosal Pathogens using UK aid from the UK Government (Project number 16/136/46) to RSH. The MLW Clinical Research Programme is supported by a Strategic Award from the Wellcome Trust, UK (206545/Z/17/Z) to SBG. The corresponding author will have full access to the study data and, together with the senior authors, will have final responsibility for the decision to submit for publication.

Disclaimer The funders had no role in study design. The funders will have no role in collection, analysis, data interpretation, writing of the report or in the decision to submit the paper for publication. The views expressed in this publication are those of the authors and not necessarily those of the NIHR or the Department of Health and Social Care.

Map disclaimer The depiction of boundaries on the map(s) in this article does not imply the expression of any opinion whatsoever on the part of BMJ (or any member of its group) concerning the legal status of any country, territory, jurisdiction or area or of its authorities. The map(s) are provided without any warranty of any kind, either express or implied.

Competing interests None declared.
Patient and public involvement Patients and/or the public were involved in the design, or conduct, or reporting, or dissemination plans of this research. Refer to the Methods section for further details.

Patient consent for publication Not required.

Provenance and peer review Not commissioned; peer reviewed for ethical and funding approval prior to submission.

Open access This is an open access article distributed in accordance with the Creative Commons Attribution 4.0 Unported (CC BY 4.0) license, which permits others to copy, redistribute, remix, transform and build upon this work for any purpose, provided the original work is properly cited, a link to the licence is given, and indication of whether changes were made. See: https://creativecommons.org/ licenses/by/4.0/.

ORCID iDs

Todd D Swarthout http://orcid.org/0000-0001-5285-7039

Robert S Heyderman http://orcid.org/0000-0003-4573-449X

\section{REFERENCES}

1 Heinsbroek E, Tafatatha T, Phiri A, et al. Pneumococcal carriage in households in Karonga district, Malawi, before and after introduction of 13-valent pneumococcal conjugate vaccination. Vaccine 2018;36:7369-76.

2 GBD 2016 Lower Respiratory Infections Collaborators. Estimates of the global, regional, and national morbidity, mortality, and aetiologies of lower respiratory infections in 195 countries, 1990-2016: a systematic analysis for the global burden of disease study 2016. Lancet Infect Dis 2018;18:1191-210.

3 Gray BM, Turner ME, Dillon HC. Epidemiologic studies of Streptococcus pneumoniae in infants. The effects of season and age on pneumococcal acquisition and carriage in the first 24 months of life. Am J Epidemiol 1982;116:692-703.

4 Ghaffar F, Friedland IR, McCracken GH. Dynamics of nasopharyngeal colonization by Streptococcus pneumoniae. Pediatr Infect Dis J 1999;18:638-46.

5 Högberg L, Geli P, Ringberg H, et al. Age- and serogroup-related differences in observed durations of nasopharyngeal carriage of penicillin-resistant pneumococci. J Clin Microbiol 2007;45:948-52.

6 Usuf E, Bottomley C, Adegbola RA, et al. Pneumococcal carriage in sub-Saharan Africa-a systematic review. PLoS One 2014;9:e85001.

7 Vallès $X$, Flannery B, Roca A, et al. Serotype distribution and antibiotic susceptibility of invasive and nasopharyngeal isolates of Streptococcus pneumoniae among children in rural Mozambique. Trop Med Int Health 2006;11:358-66.

8 Hill PC, Townend J, Antonio M, et al. Transmission of Streptococcus pneumoniae in rural Gambian villages: a longitudinal study. Clin Infect Dis 2010;50:1468-76.

9 Abdullahi O, Karani A, Tigoi CC, et al. Rates of acquisition and clearance of pneumococcal serotypes in the nasopharynges of children in Kilifi district, Kenya. J Infect Dis 2012;206:1020-9.

10 Mackenzie GA, Hill PC, Jeffries DJ, et al. Effect of the introduction of pneumococcal conjugate vaccination on invasive pneumococcal disease in the Gambia: a population-based surveillance study. Lancet Infect Dis 2016;16:703-11.

11 von Gottberg A, de Gouveia L, Tempia S, et al. Effects of vaccination on invasive pneumococcal disease in South Africa. N Engl J Med 2014;371:1889-99.

12 Hammitt LL, Etyang AO, Morpeth SC, et al. Effect of ten-valent pneumococcal conjugate vaccine on invasive pneumococcal disease and nasopharyngeal carriage in Kenya: a longitudinal surveillance study. Lancet 2019;393:2146-54.

13 Roca A, Bojang A, Bottomley C, et al. Effect on nasopharyngeal pneumococcal carriage of replacing PCV7 with PCV13 in the expanded programme of immunization in the Gambia. Vaccine 2015;33:7144-51.

14 Adebanjo T, Lessa FC, Mucavele H, et al. Pneumococcal carriage and serotype distribution among children with and without pneumonia in Mozambique, 2014-2016. PLoS One 2018;13:e0199363.

15 Flasche S, Van Hoek AJ, Sheasby E, et al. Effect of pneumococcal conjugate vaccination on serotype-specific carriage and invasive disease in England: a cross-sectional study. PLoS Med 2011;8:e1001017.

16 Jayasinghe S, Menzies R, Chiu C, et al. Long-term Impact of a "3 + 0 " Schedule for 7- and 13-Valent Pneumococcal Conjugate Vaccines 
on Invasive Pneumococcal Disease in Australia, 2002-2014. Clin Infect Dis 2017;64:175-83.

17 Moore MR, Link-Gelles R, Schaffner W, et al. Effect of use of 13-valent pneumococcal conjugate vaccine in children on invasive pneumococcal disease in children and adults in the USA: analysis of multisite, population-based surveillance. Lancet Infect Dis 2015;15:301-9.

18 Prato R, Fortunato F, Cappelli MG, et al. Effectiveness of the 13 -valent pneumococcal conjugate vaccine against adult pneumonia in Italy: a case-control study in a 2-year prospective cohort. BMJ Open 2018;8:e019034.

19 Rinta-Kokko H, Palmu AA, Auranen K, et al. Long-term impact of 10-valent pneumococcal conjugate vaccination on invasive pneumococcal disease among children in Finland. Vaccine 2018;36:1934-40.

20 Ruiz-Contreras J, Picazo J, Casado-Flores J, et al. Impact of 13-valent pneumococcal conjugate vaccine on pneumococcal meningitis in children. Vaccine 2017;35:4646-51.

21 Waight PA, Andrews NJ, Ladhani SN, et al. Effect of the 13-valent pneumococcal conjugate vaccine on invasive pneumococcal disease in England and Wales 4 years after its introduction: an observational cohort study. Lancet Infect Dis 2015;15:535-43.

22 Wiese AD, Griffin MR, Grijalva CG. Impact of pneumococcal conjugate vaccines on hospitalizations for pneumonia in the United States. Expert Rev Vaccines 2019;18:327-41.

23 Wiese AD, Huang X, Yu C, et al. Changes in otitis media episodes and pressure equalization tube insertions among young children following introduction of the 13-Valent pneumococcal conjugate vaccine: a birth Cohort-based study. Clin Infect Dis 2019;69:21622169.

24 Miller E, Andrews NJ, Waight PA, et al. Herd immunity and serotype replacement 4 years after seven-valent pneumococcal conjugate vaccination in England and Wales: an observational cohort study. Lancet Infect Dis 2011;11:760-8.

25 O'Brien KL, Dagan R. The potential indirect effect of conjugate pneumococcal vaccines. Vaccine 2003;21:1815-25.

26 Whitney CG, Farley MM, Hadler J, et al. Decline in invasive pneumococcal disease after the introduction of proteinpolysaccharide conjugate vaccine. N Engl J Med 2003;348:1737-46.

27 Melegaro A, Edmunds WJ. Cost-effectiveness analysis of pneumococcal conjugate vaccination in England and Wales. Vaccine 2004;22:4203-14.

28 Simell B, Auranen K, Käyhty $\mathrm{H}$, et al. The fundamental link between pneumococcal carriage and disease. Expert Rev Vaccines 2012;11:841-55.

29 Auranen K, Rinta-Kokko H, Goldblatt D, et al. Colonisation endpoints in Streptococcus pneumoniae vaccine trials. Vaccine 2013;32:153-8.

30 Kobayashi M, Conklin LM, Bigogo G, et al. Pneumococcal carriage and antibiotic susceptibility patterns from two cross-sectional colonization surveys among children aged $<5$ years prior to the introduction of 10-valent pneumococcal conjugate vaccine - Kenya, 2009-2010. BMC Infect Dis 2017;17:25.

31 Nunes MC, Shiri T, van Niekerk N, et al. Acquisition of Streptococcus pneumoniae in pneumococcal conjugate vaccine-naïve South African children and their mothers. Pediatr Infect Dis J 2013;32:e192-205.

32 Usuf $\mathrm{E}$, Badji $\mathrm{H}$, Bojang $\mathrm{A}$, et al. Pneumococcal carriage in rura Gambia prior to the introduction of pneumococcal conjugate vaccine: a population-based survey. Trop Med Int Health 2015;20:871-9.

33 Verani JR, Massora S, Acácio S, et al. Nasopharyngeal carriage of Streptococcus pneumoniae among HIV-infected and -uninfected children $<5$ years of age before introduction of pneumococcal conjugate vaccine in Mozambique. PLoS One 2018;13:e0191113.
34 Bar-Zeev N, Swarthout TD, Everett DB. Impact and Effectiveness of 13-Valent Pneumococcal Conjugate Vaccine on Population Incidence of Vaccine and Non-Vaccine Serotype Invasive Pneumococcal Disease in Blantyre, Malawi, 2006-2018: Prospective Observational Time-Series and Case-Control Studies [online]. Rochester, NY: Social Science Research Network, 2020. https://papers.ssrn.com/abstract= 3745169

35 King C, Bar-Zeev N, Phiri T, et al. Population impact and effectiveness of sequential 13-valent pneumococcal conjugate and monovalent rotavirus vaccine introduction on infant mortality: prospective birth cohort studies from Malawi. BMJ Glob Health 2020;5:e002669.

36 Cutts FT, Zaman SMA, Enwere G, et al. Efficacy of nine-valent pneumococcal conjugate vaccine against pneumonia and invasive pneumococcal disease in the Gambia: randomised, double-blind, placebo-controlled trial. Lancet 2005;365:1139-46.

37 Kwambana-Adams B, Hanson B, Worwui A, et al. Rapid replacement by non-vaccine pneumococcal serotypes may mitigate the impact of the pneumococcal conjugate vaccine on nasopharyngeal bacterial ecology. Sci Rep 2017;7:8127.

38 Usuf E, Bottomley C, Bojang E, et al. Persistence of nasopharyngeal pneumococcal vaccine serotypes and increase of non-vaccine serotypes among vaccinated infants and their mothers five years after PCV13 introduction in the Gambia. Clin Infect Dis Off Publ Infect Dis Soc Am 2018.

39 WHO Publication. Pneumococcal vaccines WHO position paper 2012 - recommendations. Vaccine 2012;30:4717-8.

40 Fleming-Dutra KE, Conklin L, Loo JD, et al. Systematic review of the effect of pneumococcal conjugate vaccine dosing schedules on vaccine-type nasopharyngeal carriage. Pediatr Infect Dis $J$ 2014;33:S152-60.

41 Andrejko K, Hosangadi D, Cohen O. Who technical expert consultation report on optimization of PCV impact: review of evidence and programmatic considerations to inform policy 2017;29.

42 World Health Organization. Pneumococcal conjugate vaccine (PCV) review of impact evidence (PRIME) summary of findings from systematic review [online], 2017. Available: https://www.who. int/immunization/sage/meetings/2017/october/3_FULL_PRIME_ REPORT_2017Sep26.pdf?ua=1 [Accessed 12 Mar 2019].

43 Mvula H, Heinsbroek E, Chihana M, et al. Predictors of uptake and timeliness of newly introduced pneumococcal and rotavirus vaccines, and of measles vaccine in rural Malawi: a population cohort study. PLoS One 2016;11:e0154997.

44 Tsega A, Hausi H, Chriwa G, et al. Vaccination coverage and timely vaccination with valid doses in Malawi. Vaccine Rep 2016;6:8-12.

45 Swarthout TD, Fronterre C, Lourenço J, et al. High residual carriage of vaccine-serotype Streptococcus pneumoniae after introduction of pneumococcal conjugate vaccine in Malawi. Nat Commun 2020;11:2222.

46 Lourenco J, Obolski U, Swarthout TD, et al. Determinants of high residual post-PCV13 pneumococcal vaccine-type carriage in Blantyre, Malawi: a modelling study. BMC Med 2019;17:219.

47 National Statistical Office. Government of Malawi. 2018 Malawi population and housing census main report [online]. Zomba, Malawi: Government of Malawi, 2019. https://malawi.unfpa.org/sites/default/ files/resource-pdf/2018\%20Malawi\%20Population\%20and\% 20 Housing\%20Census\%20Main\%20Report\%20\%281\%29.pdf

48 Loudon K, Treweek S, Sullivan F, et al. The PRECIS-2 tool: designing trials that are fit for purpose. BMJ 2015;350:h2147.

49 Satzke C, Turner P, Virolainen-Julkunen A, et al. Standard method for detecting upper respiratory carriage of Streptococcus pneumoniae: updated recommendations from the world Health organization pneumococcal carriage Working group. Vaccine 2013;32:165-79. 